\title{
Subjectivity, Learning and Work: Sources and Legacies
}

\author{
Stephen Billett
}

Received: 26 January 2008 / Accepted: 18 April 2008 /

Published online: 20 May 2008

(C) Springer Science + Business Media B.V. 2008

\begin{abstract}
Individuals' dispositions have long been held to direct and energise cognition in ways that shape how they experience and respond to events in the social world. Therefore, a consideration of these dispositions is likely to be helpful in understanding the inter-psychological processes between individual and social world that frame contemporary socio-cultural accounts of learning. Here, the specific concern is to elaborate the sources, legacies and potency of individuals' dispositions in the learning of occupational practice through these processes. Having reviewed ideas about the dispositional underpinnings of individuals' construal and construction of the knowledge required for work, these conceptions are exercised through illuminating the roles dispositions play in the process of constructing the knowledge required for an occupational practice (i.e. hairdressing). The study reported here combines workplace ethnography and problem-solving tasks to identify the source of these subjectivities, how they shape individuals' cognitive processes at work tasks and their learning through participation in vocational practice. In all, it identified how individuals' dispositions arise through socially-shaped life histories or ontogenies, albeit in person-dependent ways. The participants' conceptions, preferences and procedures that shape their learning and the enactment of their practice were found to be products of earlier socially-derived experiences, thereby making them personally-subjective. These personally-subjective dispositions were identified as shaping how these individuals engage in work, learning and the ongoing remaking of work activities, because they influence inter-psychological processes that comprise the immediate experiences that constitute the enactment of these activities. So, this suggests that the sociogeneses of knowledge and learning likely includes personally unique social contributions that arise through ontogeny.
\end{abstract}

Keywords Learning · Work · Subjectivity · New learning · Inter-psychological processes $\cdot$ Dispositions

\footnotetext{
S. Billett $(\bowtie)$

Faculty of Education, Griffith University, Nathan 4111 Queensland, Australia

e-mail: s.billett@griffith.edu.au
} 


\section{Subjectivity, Learning and Work}

Over the past three decades, much consideration has been given to the role that conceptual and procedural knowledge plays in human cognition, particularly within cognitive theory (e.g. Simon and Gilmartin 1973; Anderson 1982; Ericsson and Lehmann 1996). The deliberations and contributions of cognitive theory have greatly assisted understanding the kinds of conceptual and procedural goals required for competent performance and how best instruction might proceed to assist individuals to secure these forms of knowledge. However, it has been acknowledged that the character of dispositions or dispositional knowledge and its implications for cognition, learning and instruction are less well understood and are underemphasised in this literature (Perkins et al. 1993a, b; Tobias 1994). This gap in our understanding may well arise from difficulties associated with conceptualising and accessing this form of knowledge, because these dispositions are less easy to, respectively, declare or demonstrate than conceptual or procedural knowledge and are likely to be person-dependent to a high degree. This suggests that opportunities for making this form of knowledge accessible are likely to be less available than for demonstrable procedures and stateable propositions. Yet, these subjective dispositions likely underpin individuals' construal and construction of concepts and procedures, and shape how they are deployed in goal-directed activities, such as in paid work. Importantly, these dispositions likely contribute to: (i) individuals' learning and (ii) their remaking of culture. It seems that through ongoing engagement in different and overlapping forms of social practice in which they engage, these subjectively-generated and deployed dispositions are sourced and transformed throughout individuals' ontogenies or life histories (Rogoff 1990), and are socially-shaped in personally unique ways (Billett 1998, 2003). Along with their conceptual and procedural counterparts, these dispositions shape individuals' cognitive experience (Valsiner 2000) inter-psychologically: how what is encountered in interactions with the social world is construed and constructed. Indeed, some suggest that these subjective dispositions constitute the gaze through which we experience the world beyond us, including how we believe the world is viewing us (Church 2006). Therefore, more than directing energy and intentionality (Perkins et al. 1993a, b), dispositions also shape individuals' cognition through their role in categorising and ordering what is experienced and their response to those experiences. Yet, in turn, these dispositions are also themselves shaped through individuals' engagement with the social world, although the degree of reshaping is not yet understood. That is, they order individuals' conceptualisations and their enactment of procedures, and are themselves variously reinforced, reshaped or transformed through these processes in ongoing ways. Therefore, the executive properties of dispositions, such as interest, intentionality and values influence individuals' cognition. Because they are person-dependent, to some degree these dispositions are subjective.

To appraise these propositions, the contributions of personally-subjective dispositions to thinking and acting are discussed, firstly, through consideration of the concepts of interest, attitude and values. Then, the processes and findings from an investigation into the contributions of how the dispositions of nine vocational practitioners (i.e. hairdressers) construct vocational knowledge are provided to illustrate and elaborate the central role of these dispositions in the enactment of goal- 
directed activity, their sources and potential contributions to learning and the remaking of occupational (i.e. cultural) practices. In concluding, it is suggested that the development of the kinds of dispositions underpinning the enactment of work are negotiated over time and are shaped by the kinds of vocational activities that are experienced and negotiated, person-dependent ways. In all, the paper elaborates the development and role of socially-derived, yet personally-subjective dispositions in individuals' thinking, acting and learning.

\section{Nature and Contribution of Dispositions}

Cognitive theory provides helpful accounts of structures that are represented in memory and comprise networks of propositional knowledge and orders of procedural knowledge (e.g. Anderson 1982; Scandura 1982; Ericsson and Lehmann 1996). This theory proposes that how individuals conceptualise knowledge and select procedures to secure goals is shaped by and yet central to the deployment of the cognitive structures (i.e. as in learning) required for human performance in complex activities, such as paid work. This view borrows much of Ryle's (1949) classification of knowledge types into 'knowing how' (i.e. procedures) and 'knowing that' (i.e. propositions). However, while useful for understanding distinctions between procedures and concepts, the dispositions that direct and energise the deployment of procedural and propositional knowledge, their schematic linkages and organisation, and their contributions have, been less emphasised in this knowledge classification system (Perkins et al. 1993a). This becomes apparent when some behaviour cannot be adequately explained by either procedural or conceptual categories of knowledge (Martin 1970). For instance, how can: being appropriately responsive to customers in retail or restaurant settings (Billett et al. 2005), or aged care work (Somerville 2006); the service orientation and appropriateness of the level of checking and the self-monitoring required of a motor mechanic (Billett and Pavolva 2005); or the safety orientation of long-distance truck drivers (Lewis 2005) be categorised as either procedural or conceptual alone? The attributes that best capture these behaviours are the personal dispositions of attitude, affect, interest and values (Prawat 1989). Yet, these dispositions are likely to be personally subjective in their formation and deployment, making them potentially distinct in some ways in their formation from stateable concepts and demonstrable procedures, that might be seen to be derived more directly and faithfully from the social world from where they arise.

Dispositions have been viewed as individuals' tendencies to put their capabilities into action (Perkins et al. 1993a, b). This view suggests that the potency of dispositions resides in the differences between what individuals may be capable of doing and what tasks they actually undertake and how they undertake them. Such perspective might look to issues of personal motivation (Hoffman 1986) or interest (Tobias 1994) or the press of social circumstances (Barker 1968; Pace and Stern 1958) to engage learners in realising their full capabilities. Yet, beyond this quality of enacting potential, there are perhaps more fundamental roles that dispositions play in shaping how individuals construe and construct what they experience and, therefore, think and act. For example, some individuals hold implicit beliefs about knowledge, 
considering "levels of intelligence" to be fixed, while others consider their levels can be developed further (Dweck and Leggett 1988). Such beliefs shape individuals' approaches to, and attitudes about, activities associated with their agency in directing and securing their learning (Piaget 1981). These beliefs are particularly pertinent because learning is now viewed in most contemporary accounts as being a product of individuals' construction and agency, including the exercise of that agency in negotiating with the social world (i.e. inter-psychologically), rather being merely a product of external suggestion or press, as behaviouralism suggests.

Posner (1982) similarly identifies the role of dispositions in learning. He states that "the beliefs, knowledge and abilities that students bring with them into the learning setting are a product of accommodations to their environments and form frames of reference which students use to assimilate new experiences" (p. 345). Here, Posner also acknowledges the influence of earlier or pre-mediate experiences, as Valsiner (2000) describes them. Yet, the interest that comprises an agentic element of dispositions stands to energise cognition, learning and development in particular ways. A study of relatively socially-isolated learners (e.g. small-business operators), for instance, found that they exercised their agency in particular and productive ways when learning about a new taxation system, including critically appraising the worth of advice provided by others (Billett et al. 2003). That is, they deployed and exercised their personal epistemologies agentically as directed and energised by their particular interests and values.

Hence, this suggests that how individuals thinking and acting is directed, for what purposes, and with what intentionality and degree of effort (i.e. intensity) is likely shaped by their subjective dispositions (Billett et al. 2005). If this is the case, these dispositions actively shape the inter-psychological processes between the individual and the social world, are therefore generative of particular kinds of legacies or learnings, and are positioned as shaping individuals' learning and remaking cultural practices, such as paid occupations. So these subjective and personally-derived attributes are held to be central to individuals' learning and the remaking of and, potentially, transforming culture. For instance, Leontyev (1981) refers to individuals' learning (i.e. appropriation) as being the process through which each generation takes over the cultural heritage. Giddens (1984) also reminds us that social structures are reproduced and produced by the action of human subjects, and without this active remaking, societies would remain moribund. So, this production and reproduction or remaking of the cultural heritage likely arises through individuals' construal, construction and enactment of that heritage in particular circumstances and times, and for particular purposes (Billett 2003). Therefore, given learners' active role in the construction of knowledge, how they engage in particular tasks influences what they construct and learn. For example, individuals are unlikely to engage enthusiastically in acquiring knowledge that they do not value, yet others intend them to learn. Quite the opposite from what was intended may well be learnt, as individuals learn to disagree, rebuff, or contest the very knowledge that they are being pressed to learn (Hodges 1998).

Despite an appreciation of the roles that dispositions play, there remains uncertainty about the relationship between individuals' subjective dispositions and their cognitive structures (i.e. procedures and concepts). Some claim that each of the forms of knowledge referred to above are distinct, with the acquisition of one not 型 Springer 
being dependent on the others (Alexander et al. 1991). Conversely, Rohrkemper (1989) questions the separation of dispositions from other forms of knowledge as does Vygotsky (1978) who saw this separation as being a key weakness in psychological theory. Piaget (1981) suggests the relationship is one of affect that these attributes energise cognitive structures, thereby influencing, yet still being conceptually distinct from them. However, Grusec and Goodnow (1994) propose that affect and cognition need to be seen as influencing each other in ways that are not unidirectional or simple. Instead, they are likely to be bidirectional (i.e. negotiated) and complex. Torney-Purta (1992) integrates dispositions within schematic structures, and Hoffman (1986) proposes that dispositional attributes have a direct influence on cognitive structures and activities, holding that they are embedded in and underpin both knowledge 'that' and knowledge 'how'. He notes that individuals' dispositions serve variously to initiate, terminate, accelerate or disrupt information processing and shapes: "which section of the environment is processed and which processing modes operate; it may organise recall and influence category accessibility; it may provide input to the formulation of emotionallycharged schemata and categories; and it may influence decision-making" (Hoffman 1986, p. 246). Indeed, this form of selectivity has long been acknowledged as being premised in people's personal histories and experiences and shaped by their beliefs (e.g. Baldwin 1894). In all, these accounts emphasise personal dispositions, not only as a component of cognition, but also as an active and executive component of cognitive processes. Yet, given the need to understand their contributions to interpsychological processes, it is those that emphasise the bi-directional and relational interdependence between the dispositions and cognition that stand as being the most explanatory bases for what others have proposed about learning for and throughout working life (e.g. Hodkinson and Hodkinson 2004; Hodkinson et al. 2008; Somerville 2006).

Certainly, without human interest, engagement and energy, it would be difficult to view procedures or concepts as anything other than inert knowledge and their potential for enactment and further development as being moribund. Berger and Luckman (1966), whilst emphasising the social geneses of knowledge and knowing, conclude that without individual agency there would be no scientific or social progress. The way propositions are constructed, for instance, and used as goals for thinking and acting is value-laden and subjective, as are the construction and deployment of procedures. Just as procedures become compiled (Anderson 1982), concepts are chunked thereby permitting their use in ways that do not require highly engaged conscious thought. Yet, what is compiled and chunked not only carry a social legacy from the circumstances in which it was accessed, but are shaped by values and preferences that are personally particular in some ways (Billett 2003). Therefore, just as an individuals' accent represents a particular social legacy (Bourdieu 1991), the particular rendering of that legacy is shaped by personal experiences and emphases, including the preferences for words and phrases. Moreover, this proposition suggests that individuals' cognitive experience-our ways of making sense of what we experience - is inherently shaped by their interests, values and beliefs, as exercised through how we construe and construct the social world and generate its legacy through processes of compilations and conceptual associations that we then execute almost unconsciously. In this way, the legacy that comprises the 
socio-genesis of individuals' knowledge is necessarily shaped by dispositional attributes. Hence, as manifested in both the conscious and unconscious deployment of concepts and procedures, this social legacy has dispositional underpinnings that are likely to be personal and person-dependent in some ways.

Beyond the learning of new knowledge or generating new cognitive structures, individuals' deployment of concepts and procedures in goal directed activities, such as problem-solving through everyday work activities, is also associated with reinforcing existing and learning that new knowledge (Anderson 1993; Shuell 1990) i.e. learning. In this way, personally subjective dispositions are also likely to shape the on-going process of change or learning and development. Moreover, these subjective processes also engage learners in the ongoing remaking the cultural practices in which they are engaged. That is, the deployment of cognitive attributes is unlikely to be some faithful reproduction of cultural practices, but a product of individuals' interpretation and rendering of those practices at particular moments in time and circumstances, and as exercise by their dispositions. Hence, beyond the processes of transforming human cognition, as in new learning, these same processes are also involved in actively remaking practice. Consequently, existing conceptions of knowledge and learning, considerations of learning and how, for instance, individuals engage with instruction need to include and account for their dispositional underpinning. These comprise the personally-subjective value-laden nature of human thinking and acting (Billett et al. 2006). Therefore, although these dispositions can be conceptualised as a unitary entity, and open to independent analysis, these subjectivities should be viewed as inherent elements within these knowledge structures, and included in how they are represented and organised. On this basis, and in order to understand more adequately how individuals engage in activities, it is necessary to include dispositional factors centrally in deliberations about individuals' construction of knowledge in the process of their learning and also the ways in which they transform cultural practices. Yet, firstly, it is necessary to identify from where these attributes arise, so that their development, character and contributions can be more fully understood.

\section{Ontogenetic Sources of Subjective Dispositions}

As foreshadowed, dispositions arise through the negotiations between the personal and social worlds that comprise individuals' ontogenetic development or life history, seemingly making them personally subjective. Many of the accounts discussed above refer to social sources for dispositions, with experiences during individuals' life histories or ontogenies providing access to these attributes (e.g. Belenky et al. 1986; Dweck and Elliot 1983; Grusec and Goodnow 1994). Dispositions arise as individuals engage in different and overlapping social practices throughout their life histories, including those located in the home, workplaces, social groupings and schools, where individuals participate in particular ways and for specific purposes. It follows individuals' activities in these practices likely influence how they approach and engage in subsequent cognitively-demanding tasks (e.g. Dweck and Elliot 1983). Hence, individuals' valuing of a particular task will likely shape whether they enact an effortful or superficial response (Goodnow 1990). Individuals' dispositions 
appear to have their sources in their personal histories or ontogenies (Rogoff 1990) and these arise through ongoing inter-psychological negotiations between individuals' cognitive and the immediate social experience throughout life histories that are necessarily person-particular in some ways. This renders them personally-subjective to some degree. These moment-by-moment processes of cognition and learning (i.e. microgenetic development) (Rogoff 1990) serve deploy and, reciprocally, construct the dispositions that underpin thinking and acting. This occurs as what individuals know is pitted against what they experience and the process of monitoring and reflexivity that humans exercise. Therefore, the interaction between the earlier or pre-mediate subjectively-premised cognitive experience and what is subsequently encountered may provide the basis for understanding further the ongoing shaping of personally-subjective dispositions.

Nunnaly (1976) proposes a framework of interest, attitude and values upon which the sources of dispositions can be considered. Interest refers to preference for a particular activity; attitude characterises feelings about things, usually either positive or negative; and values indicates preferences for "life goals" or "ways of life". This framework is useful because it goes beyond a consideration of specific cognitive activity to include the contributions of broader goals associated with personal history ontogeny. In Table 1 and from above it is claimed that these dispositions are sourced individuals' personal histories, emphasising their subjective qualities.

To appraise the propositions advanced above, the sources and influence of personally-subjective dispositions on thinking and acting, and learning needs to be elaborated. In the next two sections, the procedures for and findings of a two-part investigation of the personal histories of the working and learning of nine hairdressers and the processes and consequences of engagement in work-related goal directed activities is reported. This investigation is used to identify the role of these dispositions in goal-directed activities and, hence, learning. In addition, they are used to provide grounded data to identify the source of those dispositions. Firstly, in Study 1, the hairdressers' professional histories, interests and preferences were identified, along with mapping the social practices in which they worked. Following that, findings from the hairdressers' responses to problem-solving activities (Study 2) are used to identify how dispositions to problem-solving activities illuminate how these hairdressers' source and construct knowledge.

\section{Method}

The data reported and discussed here are from an investigation which analysed the same vocational practice (hairdressing) in three different settings. Hairdressing was selected as a vocational activity which could be investigated in different setting, which likely provided different instances of this vocational practice. These settings were secured in three different locations in Queensland, Australia. These settings were: (i) in a provincial centre (Salon C); (ii) an outer city suburb (Salon A) and (iii) an inner city suburb (Salon F). The selection of sites was premised on gaining access to both experienced hairdressers and apprentices in the latter stages of their indenture, and to access different instances of this vocational practice. Nine subjects participated in this investigation, of which three were final year apprentices with the 
Table 1 Nunnaly's (1976) categories of dispositions and their proposed sources

Nunnaly's categories

Interest-preference for a particular activity

Attitude - feeling about things — positive or negative

Values-i) life goals - ii) way of life
Proposed sources of dispositions

Likely to be sourced in personal histories

Likely to be a product of personal history

i) Shaped by personal history

ii) Shaped by particular social environment

remaining six being experienced hairdressers. The investigation comprised two studies.

\section{Study 1}

Study 1 elicited data about the subjects' hairdressing practice, including the subjects' personal histories, preferences and interests. A goal was to identify to what degree and in what ways had the situated practice of the particular hairdressing salon, the cultural practice of hairdressing and the personal histories of individuals contribute to their vocational practice (Billett 2003). The first goal for the investigation was to understand the practice of the settings where the vocational practice occurred, an objective that demands careful systematic analysis (Salomon 1991). Hence, an ethnographic approach was adopted to analyse societal conditions, institutional settings and activity structures (Martin and Scribner 1991) because in a given setting, significant experiences will tend to co-occur in a patterned way (Barker 1968; Scribner 1984). The data were secured through rounds of interviews and lengthy observations at each of the three salons. These activities elicited: (i) a 'description of the practice' by describing the typical activities of experts and novices during busy days; determining the 'boundaries of the practice', by identifying the nature of social relationships within the social practice of the workplace (Goodnow 1990; Luria 1976) (e.g. asking questions such as those about the activities undertaken only by the expert? Which are the activities done only by the novice?). These activities included eliciting perceptions of the occupation practice of hairdressing by asking questions about the participants' perceptions and about how they think about and undertake their work, their likes, dislikes and preferences (Goodnow and Warton 1991; Tobias 1994); and asking questions about hairdressers' categorisations of clients, and how they undertake common daily tasks. Data about the work histories of the hairdressers was also gathered. In the findings reported below, the subjects are distinguished by a letter and a number (e.g. C8). The Alpha character refers to the particular salon in which they work (e.g. salon A, C or F) and a unique number from one to nine.

\section{Study 2}

Study 2 aimed to illuminate individuals' structuring and deployment of their hairdressing knowledge using analyses of protocols from the subjects' responses to a set of five ill-defined problems. These types of problems are more complex than well-defined problems, as in the former, the start and goal states are unclear, as are the operations required to secure the goal state. Thus an effect of using ill-defined 
problems is the need to elaborate more information about start state (Voss et al. 1986). These types of problems are also viewed as being particularly appropriate for an understanding about participation and learning through vocational practice as problems in vocational activities are often ill-structured, with the solver having to construct the goals and the start state (Gott 1989). Knowing why particular approaches are selected from an array of all possible representations and also the bases of that selection provides insights about how the problem has been conceptualised by the practitioner and on what bases, what solutions are offered and for what purposes (Newell and Simon 1972). For the purposes here, responses to these problems are helpful in identifying the role that personally-derived dispositions play in these processes along with situational and cultural (i.e. social) contributions to their thinking and acting. The methodological concern here is to understand in what ways personal dispositions play a role in these processes.

Accordingly, a set of five ill-defined problems, comprising a series of would-be clients with particular requests for hairdressing, was constructed and administered to the subjects in the second study. The problems were developed from observations in salons in Study 1 and from the problem-solving literature. These problems were presented to the subjects as a set of photographs of the would-be client. The subjects were handed a piece of card, with four or five attached photographs of each wouldbe client and were given a specific problem situation relating to the photographs. The photographs comprised a full frontal picture of the client, and close-up photographs of a front-view, side-view and back-view of the would-be client's head and face. When the would-be client had long hair, there was an additional photograph with the hair lifted to make the neckline visible. The subjects' responses to these problems were recorded and processed into protocols, which were then analysed and interpreted. The data presented below are those from two of the illdefined problems, the data from which best addresses the analysis referred to in this paper. These problems were presented as follows:

Problem 1 (PR\# 1) A new customer (show photographs of Robyn), with a birthmark on the side of her face, wants a haircut that is stylish, but won't reveal the birthmark. What would you do? Why?

Sub-questions/prompts

. What would you try to achieve? Why?

. How would you achieve this?

. What would you be thinking about as you are working with this customer?

. Do you have any concerns?

Problem 2 (PR\#2) A customer (show photographs of Belinda) claims that you messed up her hair last time and wants you to fix it up. What are you thinking about?

Sub-questions/prompts

. What will you do?

. What are your goals?

. How will you try to achieve your goal?

. What are your concerns? 
The protocol data permit analyses of how dispositions influence choices of goals and procedures, and also the source of those dispositions. Two studies' findings are reported separately below.

\section{Findings}

Study 1: Delineating Participants' Dispositions and Identifying Their Sources

The data from interviews in Study 1 identified areas of both commonality and difference in the subjects' personal histories, 'way-of-life' or 'life-goals' (values) and preferences (Nunnaly 1976), which shaped their approach to hairdressing. The hairdressers with responsibility for apprentices had all been apprentices themselves and, with one exception, had had work experience in other hairdressing salons. These earlier experiences had shaped their choice of the current work circumstances or 'life-goals' of four of the hairdressers, who had actively sought out their current employment in these salons. Two of the hairdressers were, in addition, realising important life goals through ownership of a hairdressing salon, in the first instance, and seeking to be respected and admired, in the second. Two female subjects, who had young children, reported being restricted in their choice of vocational activities by their part-time-work and domestic commitments. This seems indicative of how overlapping engagement in different social practice (e.g. work and family) and the subjectivities associated with each can impinge on one another, and where negotiations between the two include attitudes and values about both work and family life. Overall, the data indicate the key role that individuals' socially-determined personal histories (Greeno 1989; Prawat 1989) or ontogenetic development (Rogoff 1990) played in influencing the subjects' occupational practice. Their aspirations, interests and reasons (life-goals) for participating in hairdressing were both generated in and guided by personal histories. This was also evident in the participants' approach to vocational practice, how they sought to secure goals, how they categorised clients and planned their work, all of which are important in a goal-directed activity such as hairdressing.

\section{Preference for Practice}

Subjects' preferences for their vocational practice were indicative of how personallyconstituted dispositional factors influenced their approach to hairdressing as indicated in Table 2. Using Nunnaly's (1976) categorisation of attitudes as being either negative or positive, the subjects' preferences are depicted in this table. For example, while subjects' preferences for hairdressing activities had similarities across settings (e.g. working with clients), their preference against (i.e. dislikes) were more diverse (e.g. particular kinds of clients, particular strategies, work and nonwork life negotiations). Four subjects reported concerns emphasising how other responsibilities impinged on their work (e.g. parental role; concerns about owning and managing a business). At one salon, preference for particular techniques was reported, and each salon had a pattern of treatments which reflected its activities. In addition, the three apprentices expressed concerns about interaction with certain types of clients (i.e. older, confident mature women).

Springer 
Preference for (or against) refers to dispositions associated with clients, particular techniques, business and personal demands are illuminated here. In referring to both 'client satisfaction' and 'work with clients', the data suggest that both goals for hair cutting tasks and procedures used to secure those goals were influenced by these preferences. 'Preferences against' reported across the salons were quite diverse, being linked to factors characteristic of the salon (at Salon A-had some difficult clients) or key values (at Salon F-the hairdressing vocation and the importance of style and transforming haircuts) and to quite diverse concerns at Salon C (related to running a business; unhappy customers; and tints). These preferences related to how work tasks were conceptualised and might be enacted on the bases of the subjective premises of preferences. These data indicate that both individual and situational factors influence preference for aspects of vocational practice, yet situational factors also contributed to their personal histories, thereby shaping those preferences.

\section{Categorisation of Clients}

A values basis for the categorisation of clients was also evident in the responses. From interviews and observations, it was understood that this categorisation influenced the formation of hairdressing goals (i.e. what the hairdressing activity was to achieve) and selection of procedures used to secure goals (i.e. which particular set of procedures where to be enacted) (Billett 2003). Again, there was some consistency of client categorisation within settings. At Salon A, categorisation was value-based, drawing on personal histories and attitudes towards clients; at Salon F, on occupational and interest factors, such as freedom to be creative; at C, on concerns about treatments and the smooth running of the 'production line approach' adopted at this salon (Table 3). As, with the preferences in Table 2, these categorisations indicate a negotiation between the personal preferences of the hairdressers and factors they experienced in the salons. Differences between novices' and seniors' categorisations of clients were also evident, indicating the contributions of personal history. For example, apprentices' categorisations (A2, C9, F6), more than the seniors, were based on client attitudinal characteristics, than on attributes such as age groupings or background, as were their seniors'. The younger and less experienced hairdressers all reported uncertainty and lack of confidence in dealing

Table 2 Preference: subjects' attitudes towards their work

\begin{tabular}{lll}
\hline Sub & Preference for (likes) & Preference against (dislikes) \\
\hline A1 & Satisfying clients - chemical and colour & Awkward clients and streaking \\
A2 & Working with (some) people_cutting colour & Way some clients treat her \\
F3 & Client contact, conversation and cutting & Colouring-up-fashion cuts and weddings \\
F4 & Social aspects of work (clients and & Overlap of responsibilities between family \\
& co-workers) & and work \\
F5 & Interaction with clients_cutting & Perming and chemicals \\
F6 & Satisfying clients_creative work & Unhappy clients and those who are rude on \\
& & the phone \\
C7 & Helping clients_-self-esteem & Duties of running a business \\
C8 & Satisfying and working with clients & Unhappy clients \\
C9 & Different tasks and working with clients & Bad days-tints on tints \\
\hline
\end{tabular}


with mature women clients, which included how to engage in conversation with them and about what. This was not a cause of concern for more experienced hairdressers, who had developed these skills and were not daunted by these tasks. What this table depicts is that, while there are situational factors shaping the hairdressers categorisations of clients, there were also differences premised on their personal histories.

So, within categorisations of clients that were comparable across these settings, there were some differences (Table 3). For example, C8, who saw being "neat and tidy" as an important personal and occupational trait, classified clients by how they looked after and maintained their hair, whereas F4's classification was particularly influenced by her parental circumstances, preferring 'young mothers' as clients. F5 preferred younger people and students as this permitted him to engage in more interesting conversations and enjoy greater freedom with hair treatments, because his experience had led him to conclude that young women were more open to the kinds of transformational cuts and treatments he preferred. The categorisation of clients is an important and, perhaps formative, step in developing goal states for the hairdressing activity (i.e. goal formation) (Billett 2003). Therefore, variations in these hairdressers' responses across and within these workplace settings are perhaps illustrative of how relations among situation, person and activity (Lave 1993) influence approaches to individuals' engagement in goal-directed activities that are aligned to their conduct of, learning and remaking of the practice of hairdressing. Certainly, these differences suggest that factors outside of the press of the particular social practice shape how individuals engage in thinking and acting, and, therefore, direct and shape their learning experiences. Moreover, the references to particular instances in the participants' life experience that generated these preferences, suggest these preferences as dispositions are not ad hoc, but instead are grounded in and arise through personal histories.

\section{Planning}

Dispositions also shaped approaches to workplace planning and reflected a similar pattern of a role in the negotiation between the press of the situation and subjects' ontogenies. For example, three subjects reporting difficulties associated with effective planning, emphasised the need to be flexible to allow for contingencies, such as awkward customers, more extensive treatments and cancelled appointments.

Table 3 Client categorisation across salons

\begin{tabular}{lll}
\hline Settings & Commonality in categories & Differences in categories \\
\hline Salon A & Personal backgrounds and concerns & A1-client lifestyle (values) \\
& about clients & A2-openness/personal confidence (attitudes) \\
Salon C & Concerns about treatments and smooth & C7-what they want to spend (values) \\
& running of production line & C8-clients' mood (attitude) \\
& C9-all the same (values) \\
Salon F & Occupational and interest factors & F3-occupation and age (values) \\
& associated with clients (e.g. freedom & F4-personal standing/values (attitudes) \\
& to be creative) & F5-age and openness (values) \\
& F6-openness and sort of demands (values) \\
\hline
\end{tabular}


The significance of these subjects was their standing in these salons, as the senior hairdresser (principal participant) in each salon. Both F3 and C7, who had salon management responsibilities as well as hairdressing, emphasised an approach to planning the day's activities that avoided concerning themselves with the entire day's activities. Instead, they referred to taking work as it came along. Hence, whereas some of the hairdressers scanned the whole day's appointments first thing in the morning and commenced planning, others claimed to consider only their immediate clients, thereby reducing effortful activities associated with potentially unnecessary planning. While, such approaches might be taken as examples of more less expert ways of dealing with workplace demands, they also reflect preferences that have arisen through experience. That is, finding personally helpful ways of managing the demands of this work by engaging in the least-effortful strategies, long identified as an approach deployed by experts (Scribner 1984).

Hence, the bases for negotiating this planning was shaped by their attitudes and values that arise through their personal experience and lead to particular preferences. For instance, whereas F5 claimed to plan by intuition, F4 needed to plan ahead in order to balance her domestic and work commitments. Expressions of personal preference were clear at Salon F, where the organisation of work, with one hairdresser to each client, allowed the hairdressers there to take a different approach to planning: F3 - one at a time, keep appointments planned; F4 - need to plan for child care; F5 - 'how does the day feel"; and F6 - planning her area of speciality colours. This situation was tolerated in this salon as each hairdresser worked independently with their own clientele. Yet, this practice would not be permissible at the other two salons, where the hairdressing work is shared across hairdressers and where clients are sequentially serviced by a number of hairdressers. As a consequence of the different norms and practices for hairdressing work in these salons, the goal-directed activity of hairdressing was organised quite differently by each of the hairdressers, yet still shaped by their personal imperatives.

Hence, the goal-directed activity of planning work, comprised negotiations between the influence of the demands or press of the salons and the imperatives of personal preferences and values. Certainly, the degree by which individuals could exercise their preference (i.e. dispositional preference) was ordered by their standing in the social practices (i.e. the hairdressers' salons). Principal participants (owners/ managers) were able to utilise greater discretion of a public kind in exercising their personal preferences, than were novices. However, principal participants had to respond to different, and perhaps additional, demands of the salons' social practice because of their roles (e.g. paying wages, running a business) than did more peripheral participants. Yet, despite working in an environment that was subject to surveillance and monitoring, the novice hairdressers also reported and demonstrated being able to exercise their preferences for hairdressing goals and treatments. That is, within constraints of observable work performances within a public working space, less experienced hairdressers, whose standing was lower than managers and supervisors, were able to organise planning process in ways that suited their personal preferences. Consequently, the press of the same social practice was not uniform, nor uniformly compelling. Indeed, each hairdresser was able to exercise their personal preferences, albeit in the privacy of their decision-making or in highly public manifestations. 
In this way, there was evidence of the exercise of personally-subjective dispositions in the important process of planning vocational activities that comprised. Such planning activities also constitute instances of microgenetic development through engagement in goal-directed workplace activity activities. Whilst some of that development serves to reinforce and refine what is the individual knows and what is the preferred practice of these workplaces, there was also evidence of change arising through engaging in hairdressing activities, as is elaborated in the next section.

\section{Study 1 Summary}

When engaging in the vocational practice of hairdressing, the hairdressers' preferences for how they categorise their clients and plan their work were shaped through the interaction among the external and internal press of the salon's practices where they conducted their work and their personal histories (i.e. ontogenies) in negotiating their hairdressing practice. This included preferences for how they identified and negotiated the goals for hairdressing task, including how they how they categorised and negotiated with clients and in their planning for their work day (Table 4).

Yet, beyond illuminating the requirements for practice through observation, the interview data elaborated differences among the hairdressers' approaches to hairdressing activities, such as how they categorised and worked with clients, which they attributed to events in their personal histories. In this way, it was possible to identify particular sources of preference within subjects' ontogenetic development, such as F5's lack of training with chemical treatments, or restrictions placed on apprentices at $\mathrm{C}$ to learn what the owner-manager believed important. The knowledge used in planning was associated with dispositions and particular social practice. The sourcing found in this study strengthens claims of Perkins et al. (1993b) about the role of dispositional attributes in thinking, and Tobias' (1994) view that dispositions such as interest underpin cognitive activity. Equally, evidence is provided that dispositional factors play a role in how individuals approach the problem-solving activities, through which they remake practice, whilst learning themselves.

It is concluded from the data that the underpinning dispositional attributes of cognition can be represented as responses to experiences engaged in over time through participation in different and overlapping social practice that contribute to ontogenetic development, but in ways that are potentially personally unique (Billett 1998). These moment-by-moment problem-solving activities comprises microgenetic development and lead to particular legacies in the form of individuals' ontogenetic development that is both shaped by and the product of interaction between social experience and the hairdressers' cognitive experiences, including their subjective construction.

\section{Working and Learning Influenced by these Dispositions}

From analyses of the hairdressers' preferences the sources of these dispositions can be clustered under three headings. Those sourced from the: (i) internal press of social 
practice (Brown et al. 1989); (ii) external requirements in the forms of the cultural practice of hairdressing (Barker 1968; Pace and Stern 1958) that together comprise the social experience (Valsiner 2000); and (iii) personal histories in the form of their existing knowledge and how they construe and construct that experience (i.e. their cognitive experience). These sources are depicted in Fig. 1. Together, the data suggest, the first two contributions represent situational factors associated with the particular social practice - the hairdressing salon where the hairdressers engage in their vocational practice - with its own set of norms and values comprising the salon's culture of practice (Brown et al. 1989), that stand as a manifestation of history, cultural need and situational factors. The external environment, in the form of clients' demands and preferences, influences the approach to hairdressing problem-solving, such as willingness to take risks and the kinds of interaction with clients. Clientele characteristics provide experiences (preference for certain treatments) which are also likely to influence the hairdressers' activities. The internal press is to privilege certain approaches to hairdressing and, possibly, sanction for activities that are outside the cultural norms. For the peripheral participant (e. g apprentice), the opportunities to publicly enact practices (e.g. treatments) that are outside the norms of the setting (e.g. what we do here is....) are likely to be restricted.

Yet, in addition to these situated factors, the hairdressers' personal histories provide another source of dispositions. These histories have furnished opportunities for the development of individual sets of values and preferences through participation in unique combinations of social practices that they have participated in throughout their personal histories. Therefore, in combination, these three sources provide for the situated and ontogenetic contributions that are negotiated through individuals' subjective dispositions that underpin their construction of both hairdressing concepts and procedures.

The importance of these ontogenetically and situationally-shaped dispositions is that they underpin activities associated with the representation and deployment of knowledge and, hence, cognitive development and the remaking of the cultural practice of hairdressing. As dispositions were personally distinct in some ways among the hairdressers, their activities were likely to be influenced by that difference, in potentially personally-unique ways. In so far as the balance among the internal and external press of social practice, and personal history, are different across settings and for individuals, there are likely to be legacies for their representations of knowledge and problem-solving, as these subjective dispositions underpin their thinking and acting. These findings suggest that conceptions of cognition and learning need to account for personally-subjective and potentially idiosyncratic cognitive structures and their dispositional qualities that arise through individuals' ontogenetic development.

Table 4 Dispositional influence on subjects' approach to hairdressing

\begin{tabular}{ll}
\hline Variables & Influences on approach to hairdressing \\
\hline Preference & Setting and securing goals, including interaction with clients \\
Categorisation & Planning and organising goals \\
Planning & Approach to work activities \\
\hline
\end{tabular}




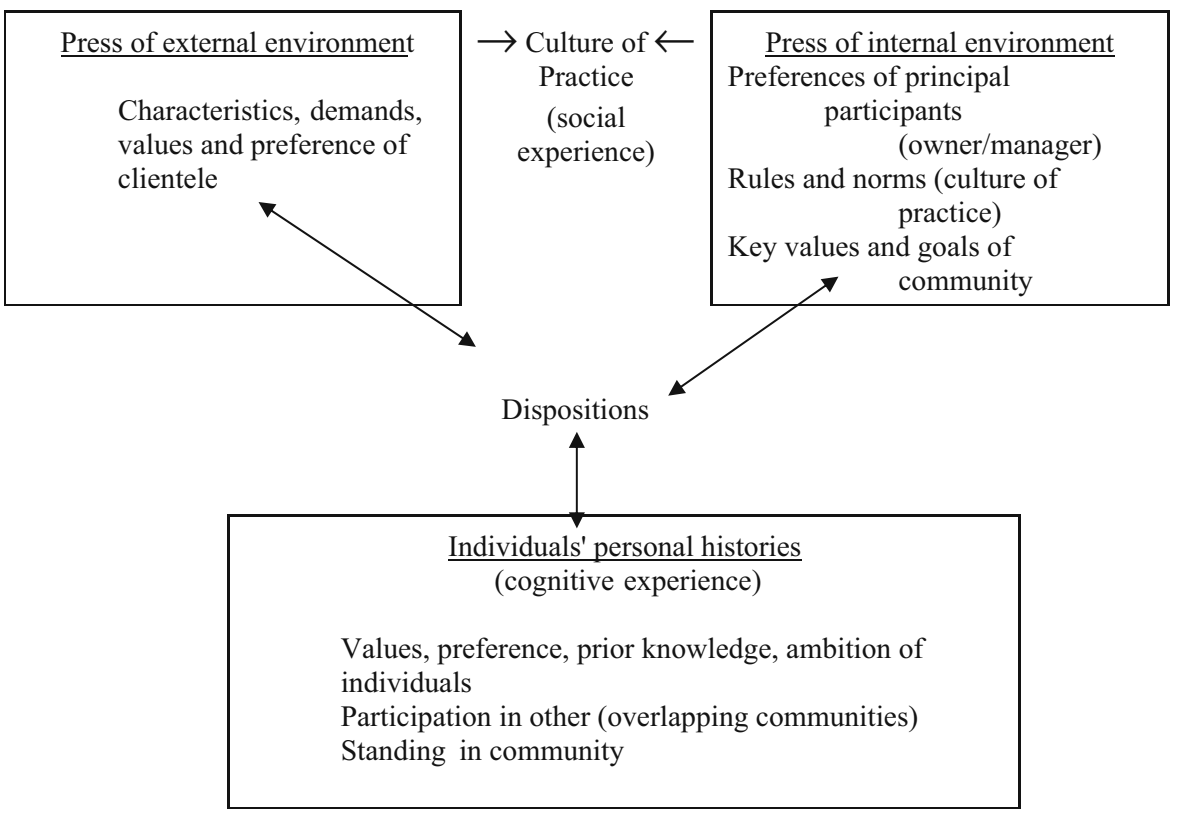

Fig. 1 Sources of dispositions (values, attitudes, interest)

This proposition, and those advanced in the earlier discussion and supported by the subjects in Study 1, are appraised further in the second study which used subjects' protocols from a series of identical hairdressing problem-solving activities.

\section{Study 2: Influence of Dispositions on Deployment of Cognitive Structures}

Findings from the protocol analyses undertaken in Study 2 elaborate how the subjects' thinking and acting were underpinned by socially-shaped personal dispositions. Concepts and procedures were identified and analysed on the basis of their being: (i) common to all sites; or (ii) common to a particular setting; or (iii) unique, or almost unique, to subjects. That is, they identified dispositions that referred to: (i) the canonical knowledge of hairdressing, that has been generated historically and in response to the cultural requirements; (ii) the manifestation of practice within the particular salon; or (iii) individual's personal history (Billett 2003). This analysis and the framework used to advance that analysis were verified by the hairdressers in follow-up interviews. In particular, they were able to identify the links between individually unique concepts and procedures with events in their personal histories and also those associated with the salons' social practice, thereby reinforcing these deductions. Moreover, inter-rater reliability procedures were used to enhance the reliability of the categorisation of data was being the product of culturally-derived canonical knowledge of the vocation (i.e. hairdressing knowledge), situational manifestation of that knowledge (i.e. the salons' norms and practice requirements) or those derived through individuals' personal histories. 


\section{Concepts which are Unique to Subjects}

Data from the two ill-defined problems introduced earlier have been used in this analysis. Data referring to concepts associated with formulating goals for a hairdressing activity involving changes to a client's hairstyle (Problem 1) and goals associated with handling a client's complaint (Problem 2) are provided. The first problem generated data on how the conceptualisation of goals resulted in the selection of specific procedures, whereas the second refers to a problem emphasising in particular the dispositional basis for conceptualising goal direct activities through which work is conducted and learning occurs.

The first of the problem tasks (Problem 1) presented subjects with a hairdressing task in which the client wanted a change of hair style; and yet was constrained by the client having a prominent birthmark on the side of her face, about which she was self-conscious. The findings consist of views about how the hairdressers' dispositions influenced both the formation of goals (i.e. concepts) and the procedures selected for their preferred treatment. Through the hairdressers' assessment of the would-be client, there was a common acknowledgment of the amount of the client's hair, the client's face shape/size and the need to consider her birthmark. These common conceptualisations represent the exercise of canonical hairdressing concepts that have developed as tools to assist this culturally derived vocational practice overtime (i.e. historically). However, different formulations of goals and preferred solutions were provided by the subjects, which reflected strong personal dispositions. For example, C7 regarded the birthmark as a problem of self-concept and proceeded to offer a severe cut which would draw attention to the side of the face (the area of the client's concern). He referred to Napoleon as having a birthmark which was matter of pride for him. In this way, the hairdresser was exercising a particular sentiment about how the client should confront the world. However, other conceptualisations emphasised the shape or size of the face (A1) or the shape of the neck (F4) as something they might incorporate in their goal for the cut. These all indicate personally-subjective construals that were identified as being person-specific.

The commonly proposed procedures (i.e. those comprising the canonical practice of hairdressing) were to achieve goals of softening the appearance around the face and leave the birthmark covered, albeit in different ways (see Table 5). On the other hand, C7's response is indicative of his beliefs about self-concept which shaped the formulation of his goal for the haircut. Note that the solutions offered in Salon F where those associated with that salon's motif about transformational cuts. In some ways, but not uniformly, the responses from three of the four hairdressers at that salon reflected versions of the kinds of cuts which that salon specialises in. So, beyond the contributions of canonical hairdressing knowledge (e.g. the general preference to shorten the hair), there was evidence also of the contributions of the particular situated practice in which the hairdressing occurred. Nevertheless, beyond these there were also differences and variations in the solutions which were product of hairdressers' dispositional preferences. Thus, the subjects' preferred goals and selected procedures appeared to provide a basis for the solution to the problem. It appears that the situated manifestation of hairdressing practice within a particular salon provided the norms for the sorts of solutions that were permissible in the salon, and the subjects selected their preferred solutions from within the array of possible 
solutions. That is, the problem space has parameters defined by the culture of practice, leaving the individual to offer solutions from within those parameters that they preferred.

In the responses to the second problem, dispositional factors were clearly evident when addressing a client's complaint, about the hairdresser having "messed up her hair" (Table 6). For instance, F3, who stated having a dislike for conflict and was a part-owner of the salon, proposed that the client may be in the wrong salon (i.e. their needs and expectations were below the trend-setting hairstyling his salon offers). Similar, the other part-owner of this salon (F4) also claimed it was the client's problem, proposing that something must be wrong in her life. These responses were contrasted to F5's desire to re-establish relationships with this client and suggested she required lots of attention and pampering. F5, it should be noted, while not a salon owner is a self-employed hairdresser who 'rents' a chair in this salon. Different again were the apprentice's (F6) concerns included her standing in the salon - the fact that she was an apprentice. So, in this salon, there were particular conceptions of this problem that lead to distinct responses. These responses reflected positions in the workplace, but also sets of dispositional factors, that were associated with the hairdressers' personal histories. The distinctiveness of dispositional nature of the response was evident in that of another salon owner. C7 interest was in resolving the problem quickly to minimise damage to the salon's reputation in the client community. C8's response was associated with self-doubt, and concerns about her reputation in the town in which she lived and worked, whereas the apprentice, C9, viewed the problem as an opportunity to learn. So, while all subjects would work seek to secure the client's satisfaction, by fixing up the problem, their goals for this and means of proceeding to address the problem were quite different.

Within the responses to this problem, attitudes (Nunnaly 1976) and personal doubts also differed across subjects and influenced how subjects approached problem-solving tasks. For example, during interviews, and in the validation process, some subjects reported concerns associated with their personal confidence, which influenced the goal they selected. This confidence determined 'how far you would go' with a particular client or, as F3 reports, "the degree that you are willing to take risks". For example, some younger hairdressers reported how uncertainty in their relationship with some mature or dominant clients influenced the selection of

Table 5 Formulating goals and selected procedures (Problem 1)

\begin{tabular}{|c|c|c|}
\hline Subject & Conceptualising goals & Selecting procedures \\
\hline A1 & Nice face-weight of hair & Straighten it with chemicals, then cut it \\
\hline $\mathrm{A} 2$ & Curly hair-difficult to visualize & Short bob-shaped cut \\
\hline F3 & Small face_-lots of hair & Give her a modern look \\
\hline F4 & Beautiful neck-lots of hair-looks like a carpet & Soften fringe and layer it \\
\hline F5 & Stylish means shorter & $\begin{array}{l}\text { Give her a piecey hair cut-long bits } \\
\text { coming in front of ear }\end{array}$ \\
\hline F6 & Curl-length & $\begin{array}{l}\text { Cut long hair into style - reduce length } \\
\text { by stages }\end{array}$ \\
\hline $\mathrm{C} 7$ & Self-image — she should learn to ignore birthmark & An asymmetrical cut, short \\
\hline $\mathrm{C} 8$ & She's all hair-small face for all that hair & Take up length and layer it around her face \\
\hline $\mathrm{C} 9$ & Change it-hold it up & Shorter bob-reduce bulk \\
\hline
\end{tabular}


Table 6 Attitudinal responses to client complaint (Problem 2)

\begin{tabular}{ll}
\hline Subject & Response \\
\hline A1 & "Where have I gone wrong?" \\
A2 & "What has she (client) done wrong?" \\
F3 & "Always take it personally" \\
F4 & "Something wrong in their (clients) lives" "something more than haircut" \\
F5 & "Control ego-get control of yourself" \\
F6 & "What did I do wrong?" \\
C7 & "Must sort out the problem" \\
C8 & "Sick feeling"- -don't know everything" \\
C9 & "Everybody's been through it—opportunity to learn" \\
\hline
\end{tabular}

hairdressing goals and procedures. This uncertainty influenced the degree of risktaking in suggesting a solution. In this way, the search or problem space was likely to be shaped by a restricted (less risky) set of options.

Some of the hairdressers reported that challenges to competence influenced their approach to clients and degree of risk-taking that even some senior hairdressers engaged in when formulating goals. However, from the protocol analyses, it was evident that sources of these attitudes were different. C7's concern was about losing the local client community's confidence, while C8's was about her personal standing in the community in which she lived. F3 reported this concern as being a challenge to his competence, as did F5. F6 viewed concerns as a product of being an apprentice - and a threat to her standing in the salon. Similarly, A1, although being a supervisor in that salon, had concerns about her security of employment and the frequency of complaints which she had to manage. It should be noted that, at this salon, the absent owner had a history of dismissing employees on the basis of customer complaints, and the clientele of this salon were observed as being prone to wanting much from the hairdressers and to complain about their hairstyles in ways that were not observed in the other salons. Thus, there was evidence that, standing in the practice influenced the dispositional basis for problem-solving, as did different external press upon that practice, such the threat of strong client complaints. The dispositional underpinnings in the responses then were shaped by the hairdressers' personal histories (life-goals and way-of-life) that comprised their earlier or premediate experiences and also those factors attributes the kinds of activities and interactions that they engaged in the salons (i.e. the immediate experience). It is in the negotiations between these that individuals' dispositions are evident in their role in shaping learning and the remaking of social practice at particular points of time and in particular circumstances. So, these legacies (i.e. learning and remaking practice) are shaped by more than concepts and procedures, it is the dispositional bases to these that shape the engagement with particular concepts and procedures that lead to particular outcomes.

\section{Sources, Influences and Contributions of Personal Dispositions}

The data analysed above suggest that the personally subjective dispositional underpinnings of conceptual and procedural knowledge influenced the hair- 
dressers' thinking and acting (problem-solving) in personally-distinct ways, albeit shaped by situational and cultural practices. Personal values appeared to determine the sort of activities that these hairdressers engaged in and how they engaged in these tasks. Both personal histories, and the internal and external press of the circumstances in which individuals engage in socially-determined activity were identifiable in the data, which supports the tentative claims that dispositions have social sources. In this way, the hairdressers' premediate experiences shaped how they construed and constructed their goals for and approach to the hairdressing task. Each of the hairdresser's ongoing experiences further shaped differences in individuals' dispositions and appeared to be the product of their construction of knowledge which was socially-derived from earlier experiences during their ontogeny.

During the validation phase of the investigation, in almost every circumstance, the hairdressers were able to account for their approach to these problem scenarios as being the product of specific premediate experiences. These experiences included treatments they had learnt in other countries (A1, C7), personal experiences and preferences (F3, F6 \& C8), or those of acquaintances (A2). These accounts were provided in quite precise and informed ways. These included experiences from when and where they had been apprenticed, worked, gone to college, etc, the sources of expectations from their values and life goals (e.g. trendy salons, other salons, friends, etc.) and images that shaped their goals for preferred hair styles (e.g. from magazines, seen in streets, styles). In sum, these practitioners could identify how particular premediate experiences shaped their representations of knowledge and problem-solving activities and had done so in ways that were, by degree, person dependent. In this way, their experiences earlier in their life histories generated a legacy that shaped how they deployed their cognitive resources in their goal-directed activities.

The analysis and deductions here propose that, for these hairdressers, personallysubjective dispositions, beyond being bases for energising individuals' capabilities, also shape the construal of experiences and how these individuals think and act, and as a consequence learn and remake their practice. From this, frameworks and categories of knowledge, such as those referring to cognitive structures, their development and deployment remains incomplete without a consideration of dispositional factors such as personal attitude, values and preference, which arise through ontogenesis. This seems a worthy advance, because despite the importance of their role in learning, these subjectively-derived attributes are still not adequately addressed within cognitive accounts, with their focus on propositional and procedural representations of knowledge. Certainly, cognitive theorists are aware of the importance of affect and dispositions (e.g. Piaget 1981; Posner 1982) and they have been the focus of much research, as on the relationship between motivation and cognition (e.g. Hoffman 1986; Piaget 1981). Indeed, earlier accounts sought to integrate dispositions in frameworks and categories of knowledge (e.g. Perkins et al. 1993a, b; Tobias 1994). Clearly, dispositions also influence whether individuals value a particular outcome enough to participate in the effortful activity required to secure the requisite knowledge. For example, Dweck and Elliot (1983) report that school students, with a performance orientation, may determine if participation in a school room activity will result in their "looking smart", which is quite a different 
goal from determining what they will learn from an activity. Consequently, determinations about engagement in activity may not be adequately addressed by the view of strategic knowledge which focuses on efficacy (e.g. what needs to be done, how and when), to the exclusion of personal dispositional factors (e.g. is it worthwhile doing, and if so how well). Strategic procedural knowledge (e.g. Gott 1989) - knowing how and when to apply knowledge-has previously been advanced as addressing value and affect. Yet, within cognitive theory, strategic or higher order procedures is usually represented in terms of the efficacy of securing goals, rather than whether the learner thinks they are worth securing (i.e. Dweck and Elliot 1983; Goodnow 1990; Tobias 1994) or whether individuals possess the personal confidence, interest or motivation to engage with what they are experiencing (Belenky et al. 1986). That is, it has not embraced the personally subjective qualities of strategic or higher order thinking. Therefore, and as demonstrated above, it is now necessary to go beyond accounts that see dispositions as merely energising cognition and exercising tendencies, to capture the character of personally-derived subjectivities and how they shape what individuals experience and come to know. Importantly, and as explained within cognitive theory, these dispositions become almost unconscious attributes as they become part of cognitive schemata that have been learnt and honed to be applied without the requirement of conscious working memory.

\section{Subjective Dispositions: Learning and Work}

In all, this paper elaborates the source, development and role of personally subjective dispositions in individuals' thinking and acting, and therefore, learning. The findings suggest that the sociogeneses of knowledge and learning includes personally unique social contributions that arise through ontogenies. Hence, the dispositions that influence how individuals think and act and remake cultural practices are personallysubjective, yet socially shaped and arise through inter-psychological processes that are shaped by both earlier (premediate) socially-derived and immediate experiences. The potency of these discussions is the role that personal histories play in shaping both cognition and culture. This comprises not only the construction of new knowledge, but also the ongoing process of development. The studies suggest that the dispositions underpinning cognitive activity are the product of individuals' ongoing participation in social practice that have contributed to legacies in the form of their cognitive experience, and how they construe and construct what they know and how they subsequently deploy that knowledge in further learning and the remaking of practice. However, not evident from this study is whether some dispositions are more or less socially-reflective, and whether some are less prone to change than others. Equally, whether there are particular kinds of experiences that lead to dispositions that are hard to change. Certainly, the evidence here is that personally-confronting experiences have a particular kind of legacy (i.e. powerful), which may also be durable. This is important because while offering insights into the genesis and transformation of dispositions, through such experiences as the interactions between the individual's ontogeny and participation in social practice are likely to engender appropriate beliefs and values that manifest themselves as 
individuals' dispositions. This adds another dimension to the inter-psychological processes between the social and the personal, and their intra-psychological outcome.

Acknowledgements The author thanks the three anonymous reviewers for their contributions to improving this manuscript, and also Professor Alison Taylor who guest edited the review of this manuscript.

\section{References}

Alexander, P. A., Schallert, D. L., \& Hare, V. C. (1991). Coming to terms: how researchers in learning and literacy talk about knowledge. Review of Educational Research, 61(3), 315-343.

Anderson, J. R. (1982). Acquisition of cognitive skill. Psychological Review, 89(4), 369-406.

Anderson, J. R. (1993). Problem solving and learning. American Psychologist, 48(1), 35-44.

Baldwin, J. M. (1894). Personality-suggestion. Psychological Review, 1, 274-279.

Barker, R. G. (1968). Ecological psychology: concepts and methods for studying the environment of human behaviour. Stanford: Stanford University Press.

Berger, P. L., \& Luckman, T. (1966). The social construction of reality. Harmondsworth, Middlesex: Penguin.

Belenky, M. F., Clinchy, B. M., Goldberger, N. R., \& Tarule, J. M. (1986). Women's way of knowing. New York: Basic Books.

Billett, S. (1998). Ontogeny and participation in communities of practice: A socio-cognitive view of adult development. Studies in the Education of Adults, 30(1), 21-34.

Billett, S. (2003). Sociogeneses, activity and ontogeny. Culture and Psychology, 9(2), 133-169.

Billett, S., Ehrich, L., \& Hernon-Tinning, B. (2003). Small business pedagogic practices. Journal of Vocational Education and Training, 55(2), 149-167.

Billett, S., Fenwick, T., Somerville (Eds.) (2006) Work, subjectivity and learning. Dordrecht, The Netherlands: Springer.

Billett, S., \& Pavolva, M. (2005). Learning through working life: self and individuals' agentic action. International Journal of Lifelong Education, 24(3), 195-211.

Billett, S., Smith, R., \& Barker, M. (2005). Understanding work, learning and the remaking of cultural practices. Studies in Continuing Education, 27(3), 219-237.

Bourdieu, P. (1991). Language and symbolic power (J.B. Thompson (Ed.)). Cambridge: Polity.

Brown, J. S., Collins, A., \& Duguid, P. (1989). Situated cognition and the culture of learning. Educational Researcher, 18(1), 32-34.

Church, K. (2006). Dressing corporate subjectivities: learning what to wear to the bank. In S. Billett, T. Fenwick, \& M. Somerville (Eds.), Work, subjectivity and learning (pp. 69-86). Springer: Dordrecht, The Netherlands.

Dweck, C. S., \& Elliott, E. S. (1983). Achievement motivation. In E. M. Hetherington (Ed.), Handbook of child psychology vol. 4, (pp. 643-691). New York: Wiley.

Dweck, C. S., \& Leggett, E. L. (1988). A socio-cognitive approach to motivation and personality. Psychological Review, 95, 256-273.

Ericsson, K. A., \& Lehmann, A. C. (1996). Expert and exceptional performance: evidence of maximal adaptation to task constraints. Annual Review of Psychology, 47, 273-305.

Giddens, A. (1984). The constitution of society. Cambridge: Polity.

Goodnow, J. J. (1990). The socialisation of cognition: what's involved? In J. W. Stigler, R. A. Shweder, \& G. Herdt (Eds.), Cultural psychology (pp. 259-86). Cambridge: Cambridge University Press.

Goodnow, J. J., \& Warton, P. M. (1991). The social bases of social cognition: interactions about work and their implications. Merrill-Palmer Quarterly, 37(1), 27-58.

Gott, S. (1989). Apprenticeship instruction for real-world tasks: the co-ordination of procedures, mental models, and strategies. In E. Z. Rothhopf (Ed.), Review of research in education. Washington, D. C.: American Educational Research Association.

Greeno, J. G. (1989). A perspective on thinking. American Psychologist, 44(2), 134-141.

Grusec, J. E., \& Goodnow, J. J. (1994). Impact of parental discipline methods on the child's internalisation of values: A reconceptualisation of current points of view. Developmental Psychology, 30(2), 4-19.

Hodges, D. C. (1998). Participation as dis-identification with/in a community of practice. Mind, Culture and Activity, 5(4), 272-290. 
Hodkinson, P., Biesta, G., \& James, D. (2008). Understanding learning culturally: overcoming the dualism between social and individual views of learning. Vocations and Learning, 1(1), $27-47$.

Hodkinson, P. H., \& Hodkinson, H. (2004). The significance of individuals' dispositions in the workplace learning: a case study of two teachers. Journal of Education and Work, 17(2), 167-182.

Hoffman, M. L. (1986). Affect, motivation and cognition. In R. M. Sorrentino, \& E. T. Higgins (Eds.), Handbook of motivation and cognition: foundations of social behaviour (pp. 236-274). New York: Guilford.

Lave, J. (1993). The practice of learning. In S. Chaiklin, \& J. Lave (Eds.), Understanding practice: perspectives on activity and context (pp. 3-32). Cambridge, UK: Cambridge University Press.

Leontyev, A. N. (1981). Problems of the development of the mind. Moscow: Progress.

Lewis, J. C. (2005). Driver competence - understanding 'hidden' knowledge through guided learning. Unpublished honours dissertation, Faculty of Education, Griffith University.

Luria, A. R. (1976). Cognitive development: its cultural and social foundations. Cambridge: Harvard University Press.

Martin, J. R. (1970). On the reduction of "knowing that" to "knowing how". In S. M. Cahn (Ed.), The philosophical foundations of education (pp. 399-409). New York: Harper \& Row.

Martin, L. M. W., \& Scribner, S. (1991). Laboratory for cognitive studies of work: a case study of the intellectual implications of a new technology. Teachers College Record, 92(4), 582-602.

Newell, A., \& Simon, H. A. (1972). Human problem solving. Englewood Cliffs, N.J.: Prentice Hall.

Nunnaly, J. (1976). Psychometric theory. New York: McGraw-Hill.

Pace, C. R., \& Stern, G. (1958). An approach to the measurement of the psychological characteristics of college environments. Journal of Educational Psychology, 49, 269-277.

Perkins, D., Jay, E., \& Tishman, S. (1993a). Beyond abilities: a dispositional theory of thinking. MerrillPalmer Quarterly, 39(1), 1-21.

Perkins, D., Jay, E., \& Tishman, S. (1993b). New conceptions of thinking: from ontology to education. Educational Psychologist, 28(1), 67-85.

Piaget, J. (1981). Intelligence and affectivity: their relationship during child development (T. A. Brown, C. E. Kaegi (Eds.). Palo Alto Annual Reviews Inc.: California.

Posner, G. (1982). A cognitive science conception of curriculum and instruction. Journal of Curriculum Studies, 14(4), 343-351.

Prawat, R. S. (1989). Promoting access to knowledge, strategy, and dispositions in students: a research synthesis. Review of Educational Research, 59(1), 1-41.

Rogoff, B. (1990). Apprenticeship in thinking-cognitive development in social context. New York: Oxford University Press.

Rohrkemper, M. M. (1989). Self-regulated learning and academic achievement: a Vygotskian view. In B. J. Zinnerman, \& D. H. Schunk (Eds.), Theory, research and practice: progress in cognitive development research (pp. 143-168). New York: Springer-Verlag.

Ryle, G. (1949). The concept of mind. London: Hutchinson University Library.

Salomon, G. (1991). Transcending the qualitative-quantitative debate: the analytical and systematic approaches to educational research. Educational Researcher, 20(6), 10-18.

Scandura, J. M. (1982). Structural (cognitive tasks) analysis: a method for analysing content. Part 1: background and empirical research. Journal of Structural Learning, 8, 101-114.

Scribner, S. (1984). Studying working intelligence. In B. Rogoff, \& J. Lave (Eds.), Everyday cognition: its development in social context (pp. 9-40). Cambridge, Mass: Harvard University Press.

Shuell, T. J. (1990). Phases of meaningful learning. Review of Educational Research, 60(4), 531-547.

Simon, H. A., \& Gilmartin, K. A. (1973). A simulation of memory for chess positions. Cognitive Psychology, 5, 29-46.

Somerville, M. (2006). Subjected bodies, or embodied subjects: subjectivity and learning safety at work. In S. Billett, T. Fenwick, \& M. Somerville (Eds.), Work, subjectivity and learning (pp. 37-52). Dordrecht, The Netherlands: Springer.

Tobias, S. (1994). Interest, prior knowledge, and learning. Review of Educational Research, 64(1), 37-54.

Torney-Purta, J. (1992). Cognitive representations of the political systems in adolescents: the continuum from pre-novice to expert. New Directions for Child Development, 56, 11-25.

Valsiner, J. (2000). Culture and human development. London: Sage.

Voss, J. F., Blais, J., Means, M. L., Greene, T. R., \& Ahwesh, E. (1986). Informal reasoning and subject matter knowledge in the solving of economics problems by naive and novice individuals. Cognition and Instruction, 3(4), 269-302.

Vygotsky, L. S. (1978). Mind in society - the development of higher psychological processes. Cambridge: Harvard University Press. 